Gefässchirurgie 2012 $17: 209-212$

DOI 10.1007/s00772-011-0975-2

Online publiziert: 16. November 2011

(c) Springer-Verlag 2011

M. Bargello · P. Langer $\cdot$ Z. Swaid $\cdot J$. Geks

Klinik für Vizeral-,Thorax- und Gefäßchirurgie, Universitätsklinikum Giessen-Marburg,

Klinik der Philipps-Universität Marburg, Marburg/Lahn

\title{
Anatomische Variante mit Ursprung der A. occipitalis aus der $A$. carotis interna
}

und eine hochgradige Stenose ( $>80 \%)$ der ACI links (NASCET).

\section{Therapie und Verlauf}

Da in der Vorgeschichte motorische Aphasien und Amaurosis fugax auftraten, stellte sich der Patient im Juli 2010 zur weiteren Diagnostik in unserer gefäßchirurgischen Abteilung vor. Da die Untersuchungen eine interventionsbedürftige hochgradige symptomatische ACIStenose links zeigten, wurde die Indikation zu Operation gestellt.

Die Operation wurde in Regionalanästhesie durchgeführt. Der Hautschnitt erfolgt entlang des Vorderrandes des M. sternocleidomastoideus mit einer Länge von etwa 8-10 cm. Nach Durchtrennung des Subkutangewebes, des Platysmas und der oberflächlichen Halsfaszie folgte die Freilegung der Gefäß-Nervenscheide der A. carotis und der V. jugularis interna. Im nächsten Schritt der Gefäßpräparation erfolgte die Unterscheidung der ACI von der ACE. Bei normalen anatomischen Gegebenheiten zeigt die ACE mehrere Gefäßabgänge zur extrakraniellen Blutversorgung. Diese sind bei der ACI hingegen nicht zu finden, da sie zugunsten der intrakraniellen Blutversorgung in der Halsregion einen direkten, unverzweigten Verlauf nimmt.

Diese sichere Unterscheidung der beiden Gefäße konnte im Fall unseres $\mathrm{Pa}$ tienten intraoperativ nicht eindeutig erfolgen, da beide Gefäße extrakranielle Gefäßabgänge aufwiesen. Nach gründlicher Präparation und Verfolgung der anatomischen Strukturen mit Ursprung und Verlauf wurde deshalb die Entscheidung getroffen, zunächst eine intraoperative digitale Subtraktionsangiographie (DSA) durchzuführen, um eine genaue Zuordnung der Gefäße zu ermöglichen. Dabei zeigte sich, dass hier eine anatomische Variante mit Abgang der A. occipitalis aus der ACI vorliegen musste.

Auf den Abbildungen des intraoperativen Situs und der CT-Angiographie ist der Abgang der linken A. occipitalis aus der linken ACI eindeutig zu erkennen (- Abb. 1, 2).

Der intraoperative Verlauf war komplikationslos, insbesondere zeigten sich keine neurologischen Auffälligkeiten. Es erfolgte eine Thrombendarteriektomie mit einer Dacron ${ }^{\circledR}$-Patchplastik der ACI sowie der A. carotis communis (ACC) mit zwischenzeitlicher Shunt-Einlage. Die DSA nach Abschluss der Gefäßrekonstruktion zeigte einen stenosefreien Fluss in der ACI und der Eingriff konnte nach 195 min beendet werden. Der postoperative Verlauf war komplikationslos. Die Wunde heilte primär, so dass der Patient in gutem Allgemeinzustand entlassen werden konnte. Auch die unmittelbar postoperative neurologische Kontrolluntersuchung zeigte sich unauffällig.

Vier Wochen später wurde der Patient erneut zu Verlaufskontrolle einbestellt. $\mathrm{Zu}$ diesem Zeitpunkt gab er keinerlei Beschwerden mehr an. Auch die FKDS konnte postoperativ keine hämodynamisch wirksamen Stenosen mehr nachweisen. Vier Monate nach der Operation wurde eine erneute Kontrolle durchge- 

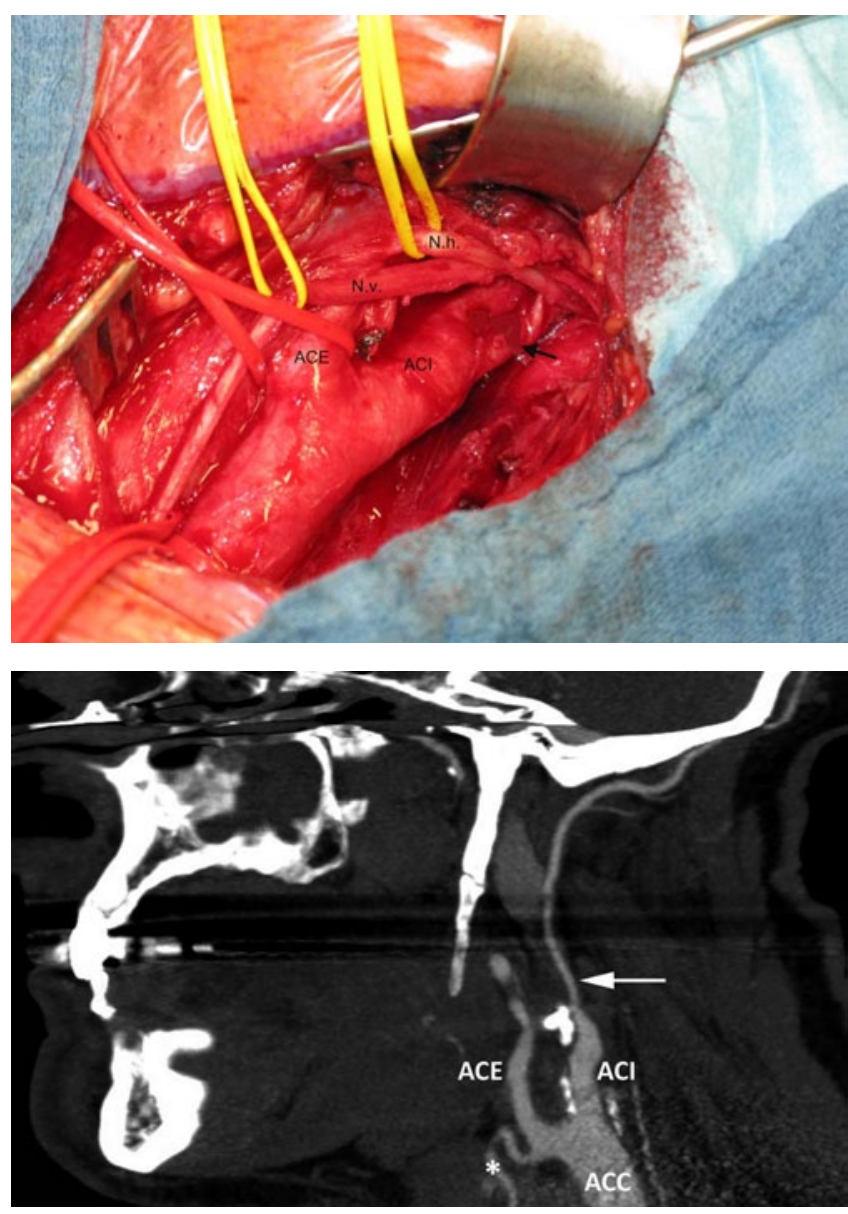

führt. Der Verlauf war weiterhin unauffällig. Es traten postoperativ keine weiteren Episoden einer motorischen Aphasie bzw. Amaurosis fugax auf.

\section{Diskussion}

Die operative Versorgung hochgradiger Carotisstenosen hat in der heutigen Zeit eine gute Prognose und gehört zu den Routineeingriffen in der Gefäßchirurgie. Wie bei allen Operationen stellen anatomische Variationen des Operationsgebietes eine Herausforderung für den Operateur dar. In unserem Fall entdeckten wir bei einem 69-jährigen Patienten während einer Carotis-TEA links bei hochgradiger ACI-Stenose eine anatomische Variation des Abgangs der linken A. occipitalis. Sie entsprang nicht wie normalerweise aus der ACE, sondern aus der ACI.

Die Äste der ACE, wie z. B. die A. occipitalis, können als Gefäßvariation aus dem zervikalen Segment der ACI entspringen $[1,3,4,7,9]$. Gerade vor chir-
Abb. $1<$ Intraoperativer Situs mit dargestellter A. occipitalis sinstra (Pfeil) aus der linken $A$. carotis interna (ACl). Zusätzlich abgebildet sind der linke N. vagus (N. v.), der linke N. hypoglossus (N. h.) und die linke A. carotis externa (ACE)

Abb. $2<$ CT-Angiographie mit Darstellung der A. occipitalis sinistra (Pfeil) aus der linken A. carotis interna (ACl). Zusätzlich abgebildet sind die linke A. carotis communis (ACC) und die A. carotis externa (ACE) mit Abgang der linken A. thyroidea superior (Stern)

samt 2866 Patienten. In 6 Fällen zeigte sich eine Anomalie der A. occipitalis, was einer Inzidenz von 0,21\% entspricht. Bei 4 dieser 6 Patienten zeigte sich ein Abgang der A. occipitalis aus der ACI (bei einem Patienten trat diese Anomalie beidseits auf). Bei einem dieser Patienten ist die A. occipitalis aus der Bifurkation der ACC entsprungen; bei einem anderen aus der A. vertebralis. Durch ihre Studie kamen sie zur Schlussfolgerung, dass eine Gefäßvariation der A. occipitalis sehr selten, aber dennoch vor geplanten Interventionen von klinisch großer Bedeutung ist [10].

Ähnlich wie in unserem Fall berichteten auch Benson [3], Bowen [4] und Aggarwal [1] über ähnliche Erkenntnisse. Benson und Hamer diagnostizierten bei einem Patienten, bei dem eine Carotis-TEA durchgeführt wurde, den Ursprung der rechten A. occipitalis aus der rechten ACI. Dies konnten sie sowohl angiographisch als auch intraoperativ nachweisen [3].

Bowen et al. beschrieben einen ähnlichen Fall [4]. Ein 67-jähriger Patient stellte sich mit symptomatischer ACI-Stenose vor. Die Duplex-Sonographie zeigte eine kleine segmentale Stenose des proximalen Gefäßteils, welche durch eine Anomalie der ACI verursacht wurde. Die darauffolgende Carotis-TEA zeigte, dass es sich bei der Anomalie um den Abgang der A. occipitalis handelte [4]. Es ist in diesem Fall also auch möglich, das die Gefäßvariation die Ursache für diese Stenose im Sinne einer Abgangsstenose war. Auch Agarwal et al. diagnostizierten bei einem 63-jährigen Patienten mit subarachnoidaler Blutung einen gemeinsamen Ursprung der A. occipitalis und A. thyroidea superior aus der ACI [1].

Anhand der wenigen Fallbeispiele

Anhand der Literatur ist die A. occipitalis der Ast der ACE, welcher am häufigsten als Gefäßvariation aus der ACI entspringt $[1,4]$. Hyrtl war 1841 einer der ersten, der von dem Abgang der A. occipitalis aus der ACI berichtete [7].

Newton und Young konnten diese Gefäßvariation 1968 angiographisch nachweisen [9]. In einer neueren Studie führten Uchino et al. entsprechende Untersuchungen auch mittels MR-Angiographie durch [10]. Diese Studie umfasste insgeund der verfügbaren Literatur lässt sich zeigen, dass trotz dieser relativ seltenen Gefäßvariationen eine genaue DuplexSonographie vor einem geplanten Eingriff von großer Bedeutung ist. Die FKDS muss dabei also nicht nur den Grad der Stenose bestimmen, sondern auch die Gefäßanatomie präzise klären. Die Kenntnis über die genaue Gefäßanatomie beugt intraoperativ bzw. während kathetergestützten Interventionen möglichen Komplikationen vor. 
Die genaue Ursache der oben beschriebenen Abgangsvarianten der A. occcipitalis ist bislang noch nicht eindeutig geklärt. Ernemann et al. erklärten diese Variation anhand der Embryologie des Carotissystems zwischen der 4. und 8. Gestationswoche [5].

\section{》) Eine genaue \\ Duplex-Sonographie ist vor einem geplanten Eingriff von großer Bedeutung}

Die proatlantale intersegmentale $\mathrm{Ar}$ terie ist eine von 4 Anastomosen zwischen den fetalen carotid-vertebrobasilaren Arterienkreisläufen [6]. Diese Anastomosen bilden sich normalerweise im embryonalen Stadium und persistieren etwa eine Woche. Anschließend, nachdem sich die Vertebralarterien entwickelt haben, bilden sich die carotid-vertebrobasilaren Anastomosen zurück. Ist dies nicht der Fall, entdeckt man gelegentlich die Persistenz dieser Gefäße. Nach der Hypothese von Lasjaunias et al. ist die A. occipitalis ein Überrest der proatlantalen intersegmentalen Arterie und kann folglich auch aus der ACI entspringen [8].

Eine aktuelle Arbeit von Arning et al. [2] beschäftigt sich mit den neuen DEGUM-Ultraschallkriterien zur Graduierung von ACI-Stenosen. Hiernach soll der Stenosegrad nach neuen Guidelines vermessen werden [2]. Bisher orientierten sich die Ultraschallkriterien an der ECST-Definition des lokalen Stenosegrades (European Carotid Surgery Trial). Da sich aber bei den radiologischen Diagnostikverfahren die Stenosegraduierung der NASCET-Definition des distalen Stenosegrades (North America Symptomatik Carotid Endarterectomy) durchgesetzt hat, wird folglich auch diese empfohlen. Neu hinzugekommen ist die Einteilung in Haupt- und Zusatzkriterien. Der Vorteil, den man sich von diesen multiparametrischen Stenosegraduierungen verspricht, ist, dass sich die verschiedenen Kriterien ergänzen und demzufolge die Stenosegrade besser abzugrenzen sind. So können beispielsweise hochgradige Stenosen in $10 \%$-Schritten graduiert werden. Arning et al. halten diese Einteilung für notwendig, um auch die Progredienz asymp-

Gefässchirurgie 2012 • 17:209-212 DOI 10.1007/s00772-011-0975-2

(c) Springer-Verlag 2011

M. Bargello · P. Langer $\cdot$ Z. Swaid · J. Geks

\section{Anatomische Variante mit Ursprung der A. occipitalis aus der A. carotis interna}

\section{Zusammenfassung}

Als Carotisstenose wird die Einengung des wichtigsten hirnversorgenden Gefäßes, der Arteria carotis interna (ACl), bezeichnet. Die Hauptursache für eine solche Stenose ist ein lumeneinengender arteriosklerotischer Plaque des Gefäßes. Das hieraus resultierende gesundheitliche Risiko besteht im Auftreten zerebraler Perfusionsstörungen, die je nach betroffenen Hirnarealen zu verschiedenen neurologischen Ausfallssymptomen führen kann, die schwere körperliche Behinderungen oder sogar den Tod zur Folge haben können. Die operative Therapie der ACl-Stenosen erfolgt i. d. R. bei entsprechender Indikation durch eine Carotis-Thrombendarteriektomie (TEA). Um das intraoperative Risiko eines Schlaganfalls gering zu halten, sind exakte anatomische Kenntnisse eine wichtige Grundvoraussetzung. Hier sind u. a. die unterschiedlichen Gefäßverläufe der A. ca- rotis externa (ACE) und der $A$. carotis interna $(\mathrm{ACl})$, welche auf Höhe $\mathrm{C} 3-\mathrm{C} 4$ aus der A. carotis communis (ACC) entspringen, zu beachten. Während die ACE in der Halsregion mehrere Gefäßäste zur extrakraniellen Blutversorgung abgibt, übernimmt die $\mathrm{ACl}$ nach unverzweigtem Verlauf die intrakranielle. Nach sorgfältiger Literaturrecherche gibt es nur wenige Berichte, die Gefäßanomalien beschreiben. Wir berichten über einen 69-jährigen Patienten, bei dem während einer Carotis-TEA wegen symptomatischer ACl-Stenose eine anatomische Gefäßvariante auffiel. Die mögliche Ursache sowie die medizinisch relevante Bedeutung werden diskutiert.

Schlüsselwörter

Gefäßvariation · Arteria occipitalis · Arteria carotis interna . Arteria carotis externa Carotis-Thrombendarteriektomie

\section{Anatomical variants with origin of the occipital artery in the internal carotid artery}

\section{Abstract}

Carotid artery stenosis (CAS) is defined as the narrowing of the carotid artery lumen and is usually caused by calcification-associated constriction through an atherosclerotic process. As a result patients are faced with a main health-threatening danger, namely cerebrovascular accidents (CVAs), which can result in variable clinical presentation depending on the affected cortical areas and potentially lead to severe physical disability or even death. With appropriate indications CAS can be treated operatively by carotid endarterectomy (CEA). In order to minimize the risk of intraoperative complications and CVAs an exact surgical anatomical knowledge is essential. This includes the origin, course and anatomical variations of the internal and external carotid arteries which arise from the common carotid artery at the level of C3-C4.
It is worth mentioning that the external carotid artery provides the blood supply to different cervical structures, whereas the internal carotid artery branches first after entering the skull. A detailed literature search revealed that there are only a few articles available describing the possible anatomical anomalies of the carotid arteries. This article presents the case of a 69-year-old patient with noticeable vascular variations, documented and observed during a CEA operation for symptomatic CAS and the medical relevance of these anatomical variations is discussed.

\section{Keywords}

Vascular abnormality - Occipital artery . Internal carotid artery · External carotid artery $\cdot$ Carotid endarterectomy tomatischer Stenosen als wichtigsten Indikator für ein erhöhtes Schlaganfallrisiko zu erfassen.

\section{Fazit für die Praxis}

Grundsätzlich ist immer mit Normvarianten der Anatomie zu rechnen, die den Verlauf einer Operation erheblich beeinflussen können. Gerade vor einer Carotis-TEA ist eine präzise präoperative Diagnostik mit Aussage zur Anatomie für einen erfolgreichen operativen Eingriff bzw. die interventionelle Therapie essenziell. So kann das Risiko einer Verletzung von varianten Blutgefäßen und 


\section{deren schweren Folgen deutlich redu- ziert werden.}

\section{Korrespondenzadresse

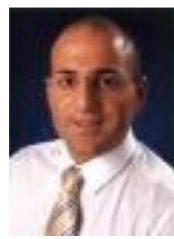 \\ Dr. M. Bargello \\ Klinik für Vizeral- \\ Thorax- und Gefäßchirurgie, \\ Universitätsklinikum \\ Giessen-Marburg, Klinik der \\ Philipps-Universität Marburg \\ Baldingerstr., \\ 35043 Marburg/Lahn \\ bargello@med.uni-marburg.de}

Interessenkonflikt. Der korrespondierende Autor gibt an, dass kein Interessenkonflikt besteht.

\section{Literatur}

1. Aggarwal NR, Krishnamoorthy T, Devasia B et al (2006) Variant origin of superior thyroid artery, occipital artery and ascending pharyngeal artery from a common trunk from the cervical segment of internal carotid artery. Surg Radiol Anat 28:650653

2. Arning C, Widder B, Reutern GM von et al (2010) Ultraschallkriterien zur Graduierung von Stenosen der A. carotis interna - Revision der DEGUM-Kriterien und Transfer in NASCET-Stenosierungsgrade. Ultraschall Med 31:251-257

3. Benson MT, Hamer JD (1988) Anomalous origin of the occipital artery from the cervical internal carotid artery. J Vasc Surg 8:643-645

4. Bowen JC, Garcia M, Garrard CL et al (1997) Anomalous branch of the internal carotid artery maintains patency distal to a complete occlusion diagnosed by duplex scan. J Vasc Surg 26:164-167

5. Ernemann U, Petersen D, Voigt K (1997) Atypischer Abgang pharyngookzipitaler Äste aus dem zervikalen Segment der Arteria carotis interna. Klin Neuroradiol 7:191-199

6. Gumus T, Önal B, Ilgit ET (2004) Bilateral persistence of type 1 proatlantal arteries: report of a case and review of the literature. AJNR Am J Neuroradiol 25:1622-1624

7. Hyrtl J (1841) Einige in chirurgischer Hinsicht wichtige Gefäßvarietäten. Med J B Ö Staates 24:421

8. Lasjaunias P, Berenstein A, Brugge K ter (2001) Surgical neuroangiography. Functional anatomy of craniofacial arteries, Vol 1. Springer, Berlin, S 165223, 370-378

9. Newton TH, Young DA (1968) Anomalous origin of the occipital artery from the internal carotid artery. Radiology 90:550-552

10. Uchino A, Saito N, Mizukoshi W, Okada Y (2010) Anomalous origin of the occipital artery diagnosed by magnetic resonance angiography. Neuroradiology. [E-pub ahead of print]
Maisch, Ristić, Seferović, Tsang Interventional Pericardiology

Pericardiocentesis, Pericardioscopy, Pericardial Biopsy, Balloon Pericardiotomy, and Intrapericardial Therapy

Heidelberg: Springer-Verlag GmbH 2011, 1. Auflage, 200 S., 85 Abb., (ISBN 978-3-642-11334-5),

Softcover, 106.95 EUR

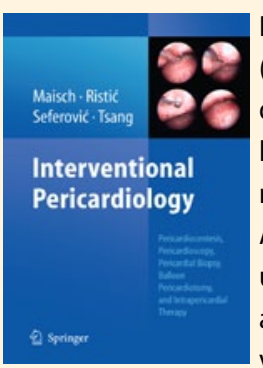
Bernhard Maisch (Marburg), führender deutscher "Perikardiologe", legt gemeinsam mit bewährten CoAutoren aus Belgrad und Vancouver eine aktuelle Publikation vor, die nahezu geeignet erscheint, ein neues kardiologisches Spezialgebiet zu begründen. David H. Spodick (Worcester, MA, USA) und Ralph Shadetai (San Diego, (A, USA), zwei herausragende Kenner der Perikarderkrankungen, haben das Werk kritisch durchgesehen und mit je einem ausführlichen Vorwort versehen. Insgesamt sind 12 Autoren - als Team - an dem Buch beteiligt; allerdings ohne dass die einzelnen Beiträge mit jeweils separatem Literaturverzeichnis namentlich zugeordnet werden können.

Hinter dem Titel „Interventional Pericardiology" verbergen sich Abhandlungen zur Perikardiozentese, Perikardioskopie, Perikardbiopsie, Ballon-Perikardiotomie und intraperikardialen Therapie. Neben bewährten Standard- und Alternativmaßnahmen, werden neue interventionelle Möglichkeiten mit indikationsbezogener Anwendung und zukunftorientierten therapeutischen Perspektiven bei Perikardprozessen erläutert, ergänzt durch instruktive Tabellen und teilweise farbige Abbildungen sowie gleichermaßen informative wie edukative Videoaufnahmen mit eingehenden Erläuterungen der Untersucher/ Autoren in Bild und Ton (CD-ROM).

Dem Register sollte das Stichwort "Complications" beigefügt werden mit Bezug zu den einzelnen interventionellen Kapiteln. Für den an Entwicklung und Geschichte der Perikardiologie interessierten Kardiologen, ist die Lektüre des historischen Kapitels zu Beginn eine besondere Freude (von Hippokrates über
Kaiserin Sissi bis Cournand und den Autoren der vorliegenden Schrift...).

Fazit: Es handelt sich um ein außergewöhnliches Buch, das eine sehr spezielle nosologische Entität zum Thema gewählt hat, die bislang noch nicht die notwendige Aufmerksamkeit - allenfalls in den USA - gefunden hat. Die Autoren heben mit Recht hervor, dass bei Perikarderkrankungen nicht-invasive diagnostische und therapeutische Prozeduren an erster Stelle stehen. Das Buch richtet sich an alle, die mit perikardialen und myokardialen Erkrankungen wie auch innovativer Perikardiologie befasst sind; dies schließt Molekularkardiologen, Immunologen ebenso ein wie Elektrophysiologen und Herzkatheterspezialisten. „Interventional Pericardiology" sollte ungeachtet des respektablen Anschaffungspreises in keiner kardiologischen Bibliothek mit dem Anspruch auf der Höhe der Zeit zu stehen, fehlen.

Professor Dr. Berndt Lüderitz (Bonn) 